\title{
Europe et communication : le labyrinthe
}

Nicole Denoit et Béatrice Galinon-Mélénec

\section{OpenEdition}

Journals

Édition électronique

URL : http://journals.openedition.org/communicationorganisation/2309

DOI : 10.4000/communicationorganisation.2309

ISSN : 1775-3546

\section{Éditeur}

Presses universitaires de Bordeaux

\section{Édition imprimée}

Date de publication : 1 mai 2000

ISSN : 1168-5549

\section{Référence électronique}

Nicole Denoit et Béatrice Galinon-Mélénec, «Europe et communication : le labyrinthe », Communication et organisation [En ligne], 17 | 2000, mis en ligne le 27 mars 2012, consulté le 20 avril 2019. URL:

http://journals.openedition.org/communicationorganisation/2309; DOI : 10.4000/ communicationorganisation.2309

Ce document a été généré automatiquement le 20 avril 2019.

(c) Presses universitaires de Bordeaux 


\title{
Europe et communication : le labyrinthe
}

\author{
Nicole Denoit et Béatrice Galinon-Mélénec
}

1 Le 25 mars 1957, à Rome, six états sont présents pour signer le traité instituant la Communauté économique européenne : les trois pays du Bénélux, la France, l'Italie et la république fédérale d'Allemagne. À cette occasion, le chancelier Konrad Adenauer déclare : «C'est seulement en se mettant ensemble dans un espace économique commun que les pays de l'Europe peuvent, à long terme, redevenir et rester compétitifs ». Bien que les objectifs économiques n'épuisent pas à eux seuls les ambitions du traité, le primat de la dimension économique marque l'ensemble du processus : la construction commence par la définition d'un marché unique concrétisé successivement par la libre circulation des marchandises et le désarmement douanier, puis la libre circulation des personnes, des services et des capitaux. La construction de ce marché unique est ensuite passée par le choix d'une monnaie européenne. Le fait qu'en décembre 1995, les quinze la baptisent «Euro» montre que les institutions européennes basent leur politique communicationnelle sur un parti pris sémantique du rappel de la localisation. En abandonnant l'Ecu - qui s'inscrivait lexicalement dans une histoire de l'ordre du monétaire - pour l'Euro, les institutions européennes replacent la logique économique dans une problématique d'affirmation de l'identité « Europe » et de ses contours.

2 En 1997, en pleine préparation de l'union monétaire et du lancement de l'Euro, nous avions lancé un premier appel à articles en vue de la coordination de ce dossier. L'Euro était alors devenu un enjeu de communication pour les entreprises. Les entreprises qui réagissaient le plus vite en espéraient un avantage comparatif non seulement en termes de parts de marché mais aussi en termes d'image. Nous posions aussi la question de l'enjeu du rôle d'interface communicationnel des États en tant que relais des institutions européennes. Dans ce contexte, souhaitant délimiter un objet transversal et partiel en vue de nous prémunir contre une trop grande disparité dans les angles d'approche des contributions, nous proposions la mise en place de l'Euro comme domaine d'application des analyses de politique de communication. 
3 Le peu de contributions proposées par les chercheurs nous a conduites à renouveler l'appel à articles, axant davantage le propos sur la volonté de l'Europe de créer un espace intermédiaire entre l'espace monde et l'espace Etat-Nation. La visée transnationale de l'identité européenne trouvant des résistances quotidiennes dans les réalités disparates des pays concernés, il s'agissait de mesurer l'importance de ces disparités et d'évaluer si une communication valorisant la logique unifiante du transnational pouvait contribuer à créer un espace qui rende possible le dépassement des différences. Nous proposions de penser cette dimension en nous interrogeant sur tous les éléments permettant de cerner comment s'opérait la prise de conscience d'une limite spatio-culturo-économique entre l'Europe et les cultures externes. Les contributions rassemblées pourraient s'articuler autour de quatre points :

- Une communication fonctionnelle et instrumentale contestée.

- Eurosceptiscime vs europtimisme.

- Des micro au macro limites spatio-culturelles.

- Le droit au secours de la communication.

\section{Une communication fonctionnelle et instrumentale contestée}

9 Françoise Massart-Pierard, analysant les circonstances de la réaction de rejet qui s'est manifestée lors du traité de Maastricht, ouvre ce dossier en montrant la place essentielle de la communication dans la construction de l'Europe. Dès ce premier article, on peut prendre la mesure des difficultés à proposer une vision de la construction européenne qui emporte l'adhésion du grand public. Les erreurs de stratégie de communication abondent. Ainsi la Commission européenne propose une vision fondamentalement technocratique inspirée avant tout par la rationalité néofonctionnaliste. L'analyse permet ici de comprendre les résistances qu'une telle politique de communication a pu développer et aussi l'urgence des mesures prises pour améliorer, après le traité de Maastricht, l'image de la Communauté européenne auprès des citoyens. La recherche d'une meilleure intelligibilité de la législation communautaire dans le cadre du renforcement du caractère démocratique du processus de décision, l'institutionnalisation $\mathrm{du}$ dialogue avec les groupes d'intérêt pourraient faire croire à une communication interactive au cœur des nouvelles orientations de la politique européenne. Cependant, l'auteur s'oriente vers le constat d'une stratégie communicationnelle de la Commission s'établissant sur une relation de puissance qui instrumentalise le citoyen.

10 Également sensible au déficit de la communication européenne, Christian Mesnil la perçoit entre «contrainte, séduction et débat ». Son propos complète l'analyse de cet écart d'incompréhension "qui semble se creuser entre les instances communautaires et les populations concernées ». L'article tente de repérer et d'analyser les tensions perceptibles dans les échanges à l'intérieur de l'Europe, notamment ceux qui sont relatifs au chassé-croisé de discours nationaux et communautaires, à une absence structurelle de visibilité, à l'inégale efficacité d'une communication institutionnelle. Dans cette analyse, en écho à celle de Françoise Massart-Pierard, l'accent est mis sur les dangers de l'hégémonie d'une communication techno-libérale. Cependant, l'auteur montre aussi que le déficit de communication des institutions européennes tient en partie à leur structure 
et à leurs missions qui correspondent mal aux exigences médiatiques actuelles : dans un organisme communautaire la nécessité de la concertation et du consensus empêche une réactivité médiatique et impose des propos nuancés respectant la coexistence de points de vue différents. Christian Mesnil souligne la difficulté à « passionner le grand public sur des dossiers techniques nuancés et impersonnels». Il y aurait donc ici quelque fatalité difficile à contourner. L'analyse s'arrête sur le risque d'un déficit de communication entrainant l'incompréhension, le contresens dans l'opinion et contribuant ainsi au développement de positions non seulement défiantes à l'égard de la politique européenne mais d'un conservatisme sectaire et rétrograde. Au-delà des dangers signalés, des propositions sont avancées. Elles insistent rappelant la thèse de Dominique Wolton - sur la nécessité de «ralentir la vitesse de l'information pour renforcer le lien social et le partage, pour réhabiliter la politique face à une communication omniprésente en réseaux hypertechnicisés ». L'analyse de Christian Mesnil montre bien qu'une communication omniprésente ne peut pallier le déficit de légitimité des institutions européennes. Il s'agit d'abord d'engager une réflexion profonde sur une construction européenne démocratique, sur la base d'un véritable débat entre citoyens-acteurs.

\section{Euroscepticisme ou europtimisme?}

11 La réflexion d'Éric Dacheux part également de cet «euroscepticisme galopant » et se propose d'évaluer la validité des explications avancées pour ce phénomène : l'insuffisance de la politique de communication mise en place par la Commission Européenne. Décrivant la diversité des moyens mis à la disposition de la DG X (Direction Générale " audiovisuel », information, communication culture), il souligne par là même les erreurs d'utilisation. Ainsi, cette croyance en la toute puissance symbolique de la télévision pour développer le sentiment d'appartenance, ou le mythe de l'avènement d'une société de l'information par un accès aux nouvelles technologies égal pour tous. À ce propos, l'auteur explique fort bien le décalage entre l'information et la participation politique c'est-à-dire l'implication des citoyens dans la construction européenne. Autre erreur: concevoir l'Europe en évolution comme un " produit » marketing fini auquel on pourrait adapter le principe de segmentation et de séduction en totale contradiction avec la complexité d'une réalité européenne, humaine, géographique, historique qui n'a rien à voir avec un marché unifié. Nous retiendrons, de la longue liste des problèmes pouvant expliquer ce qu'Éric Dacheux appelle catégoriquement "l'inefficacité de la politique de communication de la Commission ", un bilan très sévère dans les termes qu'il reprend au juriste Dominique Rousseau: " en multipliant les institutions, en s'entassant les uns sur les autres, en se modifiant les uns les autres, traités et conventions ont donné naissance à un " monstre » constitutionnel incompréhensible par les citoyens et peut-être même par les initiés ». Finalement, l'analyse d'Éric Dacheux rappelle ce à quoi aboutissaient, en d'autres termes, Françoise Massart-Piérard et Christian Mesnil : la communication ne peut tenir lieu d'un projet politique identitaire soutenu par les citoyens des pays membres. Or ce projet est particulièrement difficile à concevoir dans le contexte de la crise de confiance dans la politique. Face à cette situation, Éric Dacheux rappelle deux perspectives d'avenir pour l'Europe, celle de Jürgen Habermas qui prévoit, après l'effondrement de l'Etat-nation, l'avènement d'une démocratie deliberative, un « patriotisme constitutionnel » distinct de «l'identité culturelle». À l'opposé de cette vision normative, la perspective d'avenir qu'envisage la Commission européenne repose sur « un espace public virtuel » qui, via 
Internet, viendrait pallier le déclin d'une démocratie représentative. Une troisième hypothèse, avancée par l'auteur, est celle d'une «interculturalité européenne venant s'ajouter et non se substituer aux identités collectives existantes». L'article d'Éric Dacheux propose donc une analyse critique de la situation actuelle en même temps qu'une réflexion novatrice sur l'identité culturelle de l'homme moderne «forcément plurielle», construite par «l'ensemble des processus conduisant à l'appropriation par l'individu de la culture de son pays ». Dans cet esprit, l'article souligne l'intérêt des rencontres associatives européennes qui contribuent à l'émergence d'espaces publics européens autonomes, éphémères, non institutionnels.

12 En affirmant que c'est une erreur, face à l'euroscepticisme, de céder à la tentation de proposer une réponse technologique à des problèmes politiques qu'on ne sait pas résoudre, Éric Dacheux anticipe les propos d'Anne-Marie Autissier et de Jean-Michel Djian dans le domaine culturel. En effet, leur contribution dans ce dossier vise à montrer que ni l'alibi culturel ni aucun artefact communicationnel ne peuvent masquer l'absence d'un véritable projet politique européen produisant du sens pour chaque citoyen.

13 L'article de Lise Vieira réaffirme la nécessité pour l'Europe d'une élaboration identitaire à visée unifiante. On trouve donc une continuité dans l'objectif à atteindre, cependant sa position est très différente de celle des analystes précédents dans la mesure où elle reprend à son compte, en l'explicitant, l'hypothèse de la Commission Européenne selon laquelle l'unité de l'Europe pourrait trouver une solution dans «la conception et la diffusion de produits d'information sur les réseaux et en particulier sur Internet». Lise Vieira considère en effet que l'information est «désormais un enjeu économique et stratégique considérable au plan mondial ». Sa recherche sur l'édition multimédia met en évidence les dangers d'une concentration des grands trusts internationaux préoccupés seulement de rentabilité. L'auteur développe les mesures d'incitation à l'édition électronique qu'encourage la Commission Européenne car elle est elle-même convaincue que « la diffusion de la connaissance peut en partie remédier à la montée de la violence et des intégrismes à condition que les industries culturelles, notamment l'édition électronique intègrent les notions de coopération et de muticulturalisme ». Lise Vieira développe une position optimiste quant au rôle des nouvelles technologies d'information et de communication dans la constitution de l'identité européenne, convaincue que « l'attitude active de l'utilisateur fait du réseau à la fois un catalyseur du lien social et un outil de la pluralité des approches et des cultures ».

\section{Des micro aux macro limites spatio-culturelles}

14 Avec la perspective de recherche ouverte par Paul Rasse, on est loin des nouvelles technologies et de «l'espace public virtuel» via Internet dont rêve la Commission Européenne. L'auteur aborde la question de l'Europe et de la communication à travers les identités culturelles locales dans leurs relations avec les phénomènes de mondialisation. L'approche des réalités régionales prend appui dans son article sur une connaissance à la fois historique, géographique et anthropologique du contexte méditerranéen. Oubliant les nouvelles technologies, c'est aux éléments naturels que l'on revient, dans leur spécificité territoriale, pour interroger la possibilité de construire une unité culturelle européenne. Soulignant en quoi le concept d'aménagement du territoire, «planifié par le haut par l'État technocratique dans la perspective de réalisations gigantesques " se différencie du développement local qui vient de la base sans perspective réelle de 
rentabilité. Paul Rasse montre que « la mémoire régionale reste la source de l'identité » et que l'Europe de la diversité s'appuie sur la dynamique des identités régionales. Dans ce mouvement identitaire, "la Méditerranée occupe une place de choix " que l'auteur analyse en précisant les risques d'une standardisation touristique qui mépriserait l'histoire et le patrimoine. L'article montre ce qu'on peut attendre d'un mouvement identitaire bien compris à l'échelon européen. « Est-ce trop attendre de la communication moderne qu'elle favorise ni le renfermement tribal, ni l'universalisme abstrait, mais la pluri-appartenance et les identifications tournantes?»

C'est également la diversité des cultures qui intéresse Joanna Nowicki mais davantage dans la perspective de comprendre comme se perçoivent les citoyens de l'Europe occidentale et ceux du centre et de l'Est. Nul doute,peut-on dire grâce à son analyse, qu'un occidental n'a pas à justifier de son européanité mais qu'un centre ou un est européen ne jouit pas de ce privilège.

Il se sent appartenir à une Europe « cadette ». À situer l'Europe occidentale au-dessus, on lui interdit toute défaillance, tandis qu'on peut craindre pour l'« Europe Médiane » une trop grande tendance à ne se concevoir qu'à travers le modèle de l'Europe occidentale. Soulignant la nécessité pour l'Europe occidentale d'intégrer l'expérience de l'Autre Europe, au contraire de se limiter à l'euphorie du progrès technique, J. Nowicki rappelle les richesses d'un cosmopolitisme bien compris, humain et tolérant. On retrouve dans cet article le balancement déjà décrit entre le local et l'universel car le cosmopolitisme centre européen peut, selon l'auteur, être considéré comme l'expression locale spécifique de l'universalisme occidental.

\section{Le droit au secours de la communication?}

17 C'est enfin la perspective juridique qui ferme ce dossier, celle d'un « juriste intéressé au droit européen (qui) vient s'aventurer dans la science de l'information et de la communication ", persuadé qu' « améliorer la communication permet d'appréhender un processus organisant de l'union européenne, facteur essentiel au sens propre à prendre en considération pour un droit qui reste un droit de la construction de l'identité européenne ». L'approche juridique de Loïc Grard fait apparaître l'Union Européenne comme un « objet juridique non identifié », « une nouvelle forme d'organisation en train de s'inventer sous nos yeux par la volonté d'une quinzaine d'états ». À partir du principe de transparence et en réunissant « les perspectives de la science juridique et de la science de l'information et de la communication ", l'auteur examine les moyens mis en œuvre pour corriger le hiatus entre l'institution et le particulier, ceci depuis le début des années 90 tandis que s'impose en France un grand mouvement de transparence entre administration et administrés. Cependant la notion de transparence dans la politique de la Communauté Européenne est encore insuffisamment ressentie. C'est au contraire l'impression d'opacité qui domine. Par ailleurs, l'analyse montre bien que si « la culture du secret recule », le droit d'accès à l'information détenue par la Communauté s'arrête là où commence la protection du secret des affaires. La difficulté et la réussite du principe de transparence résident dans l'aptitude à concilier liberté d'expression, intérêt du citoyen à accéder aux documents et intérêt de l'institution à préserver le secret, toute décision devant par ailleurs être explicitement motivée. On devrait s'acheminer vers une «mise en place d'une législation qui impose la transparence par l'accès à l'information pour tous mais aussi par la clarté et l'intelligibilité du droit qui constitue l'exigence 
démocratique que la Communauté européenne s'efforce de servir ». On comprend avec l'approche de Loïc Grard que légiférer et communiquer ne sont plus des démarches séparées mais qu'elles se nourrissent mutuellement de leurs propres exigences.

\section{Conclusion}

18 En définitive, les contributions rassemblées dans ce dossier renforcent, pour la plupart, l'idée que les institutions européennes utilisent une communication technocratique, descendante et fonctionnelle qui instrumentalise le destinataire (le citoyen, l'entreprise) de l'information. L'observation d'un développement croissant des nouvelles technologies ne modifie pas ce point fondamental. Certaines contributions espèrent que la circulation de l'information pourra être organisée pour permettre une attitude réactive du destinataire final. Mais les institutions sont-elles prêtes à gérer les informations ascendantes et les NTIC peuvent-elles sérieusement espérer servir le renforcement d'une intercuturalité européenne? Certaines contributions partent des identités culturelles locales et des spécificités territoriales et rappellent que l'Europe repose sur le dynamisme des identités régionales. Les unes et les autres ne se posent pas la question de la limite du territoire Europe. Est-ce parce que le sentiment d'appartenance n'est pas mis en cause ? Parce que l'Europe correspond administrativement à un contenu en perpétuel changement? Parce que forger des normes consensuelles est tout aussi délicat? Le droit semble d'un faible secours face à un objet juridique aussi mal identifié. Le juriste suggère que la législation la plus pertinente pourrait être celle qui porterait sur les processus communicationnels internes qui imposeraient une certaine « transparence ».

Il est aussi possible de poser la question de la limite entre l'interne et l'externe. Certes, elle repose administrativement sur les décisions des institutions européennes. Elle se réalise, humainement, sur la prise de conscience par ses membres du sentiment d'appartenance ou d'exclusion. Ce dernier peut s'ancrer à partir de l'importance de la communication persuasive nécessaire pour se faire reconnaître « européen ».

Renan définissait l'Europe comme "une confédération d'États réunis par une idée commune de la civilisation ». Nourrie par l'Histoire, elle peut-être renforcée par les médias ${ }^{1}$. Ils jouent un rôle clef dans le processus d'identification d'un territoire par les membres qui le composent ${ }^{2}$.

21 En tous les cas, la multiplication des échelons régionaux, nationaux, supranationaux, le caractère changeant de l'espace englobant, la mise en place de réseaux de communication qui «déterritorialisent» la communication - pour créer leurs nouveaux territoires posent la question du type de communication à mettre en œuvre. « Il y a une conjonction entre le souci des Régions, ou des citoyens dans les régions, d'avoir plus à dire et de participer et une extension des compétences communautaires. Cela appelle un réexamen des liens et des structures de dialogue entre différents niveaux d'actions concernés, c'està-dire entre les Régions, l'État et l'Union ${ }^{3}$ ».

Pour notre part, nous dirons que les réseaux de communication recomposent des territoires propres à leur objet. L'Europe administrative impose une carte de "son » territoire. Elle le fait dans une vision centralisatrice et en ignorant largement la réalité plurielle du « terrain » et l'entrecroisement des espaces. Faut-il y voir une volonté ou une incapacité à concevoir une communication alternative? Le dossier laisse la question en 
suspens et offre l'opportunité d'une synthèse ultérieure de recherches dans un domaine riche en problématiques encore inexplorées.

Communiquer pour organiser un espace dans lequel chacun puisse trouver son appartenance tout en sentant préservée sa différence. Construire une politique consensuelle mais ferme, qui propose des mesures nécessaires mais sans précipitation, de façon à ce qu'un lien social, une nouvelle citoyenneté européenne puissent s'y nourrir. Faire et faire savoir certes, mais dans un fonctionnement de type démocratique qui se garde d'une information par trop impérative qui générerait le rejet des décisions institutionnelles à l'échelon européen. Autant de convictions partagées par les auteurs qui ont bien voulu apporter leur contribution à ce dossier, cependant que les analyses proposées ici divergent par la diversité des préoccupations et des méthodologies. Divergences fructueuses car éclairant sous de multiples aspects la réalité européenne. Regards croisés et perspectives qui permettent de mieux saisir le rôle que peuvent et doivent impérativement jouer les sciences de l'information et de la communication dans la construction d'une identité européenne.

\section{NOTES}

1. Cette problématique ayant déjà fait l'objet d'une publication scientifique collective de nos collègues en Sciences de l'Information et de la Communication, elle ne sera pas abordée dans le dossier. Cf. Miège B. (Ed.), Médias et communication en Europe, Grenoble, P.U.G, 1990

2. A propos d'Arte, lire l'article de Nahima Vianna «Culture et télévision en Europe : le cas d'Arte ».

3. Mabileau, A., (1996), « La région, relais entre l'Europe et le citoyen », Actes de la table ronde ${ }^{\circ}$ 4 , 3ème rencontres de la communication territoriale : La communication des régions. 